\title{
Per una tipologia della metatestualità integrata nel Peccato di Giovanni Boine
}

\author{
Sveva Frigerio \\ Università di Ginevra
}

\begin{abstract}
Il peccato (1913-I4) di Giovanni Boine è per contenuti e forma un'opera audace, che racconta l'amore fra il protagonista e una suora adottando la tecnica del flusso di coscienza e iscrivendola in una struttura densa di incisi. Nel presente contributo si propone un'analisi linguistico-stilistica di alcune di queste formulazioni parentetiche, a partire da un modello più generale di catalogazione delle strategie metatestuali d'autore.
\end{abstract}

Keywords: Giovanni Boine, inciso, parentesi, flusso di coscienza, metatestualità

Pubblicato per la prima volta fra il 1913 e il 19I4, Il peccato di Giovanni Boine (I887-I9I7) è per contenuti e forma un'opera audace, che intorno ad un sottile filo narrativo sviluppa una componente riflessiva densa, talora intrecciata alle analessi, e articolata secondo una complessa gerarchia. ${ }^{1}$

Suddivisa in tre sezioni estremamente compatte e all'incirca equivalenti («Il limbo», «La qualunque avventura», «Il tormento»), l'opera racconta dell'innamoramento fra il protagonista, un giovane intellettuale fresco di studi, e una suora. Dopo lo scandalo suscitato in paese, la donna abbandonerà la vita monastica per sposare il protagonista, che parallelamente all'avventura sentimentale affronta l'entrata nella vita adulta, l'assunzione di responsabilità, il passaggio all'azione, che si contrappone alla tradizione

I L'opera appare inizialmente sulla «Riviera ligure»: i numeri 22 (ottobre I9I3) e 26 (febbraio I9I4) accolgono rispettivamente la prima e la seconda parte, mentre i numeri 29 (maggio I9I4) e 3 ( (luglio 19I4) accolgono la terza. Viene pubblicata per la prima volta in volume nei «Quaderni della voce» (XXIII, maggio I9I4), insieme a due altre prose (La Città e Conversione al Codice), senza varianti di rilievo ma con eliminazione di parte dei refusi presenti nell'edizione in rivista, mentre altri permangono anche nella seconda edizione in volume (Firenze, Soc. An. Ed. «La Voce», 1922). Una terza edizione è curata da Mario Novaro (Modena, Guanda, 1938), con lacune solo parzialmente ripristinate nell'edizione successiva (Il Peccato e le altre Opere, Parma, Guanda, 1971, con un Ritratto di Boine di Giancarlo Vigorelli e un Ricordo di Mario Novaro). Segue l'edizione critica curata da Giulio Ungarelli (Torino, Einaudi, 1975), dalla cui Nota al testo (pp. XXIII-XXV) sono tratte le informazioni qui riportate. Ad essa fa riferimento con minime varianti anche la successiva edizione curata da Davide Puccini (II peccato - Plausi e botte - Frantumi - Altri scritti, Milano, Garzanti, 1983). Fra le edizioni recenti si segnalano Il Peccato, a c. di Ugo Perolino (Bologna, Millennium, 2003) e Il peccato ed altre cose, a c. di Fabio Barricalla, con un saggio di Franco Contorbia e uno scritto di Giuseppe Conte (Mallare, Matisklo, 2014), che accosta al Peccato le due prose pubblicate con esso nel 19I4. Per un inquadramento generale si rinvia a Mario Costanzo, Giovanni Boine, Milano, Mursia, I96I e a Renato Minore, Boine, Firenze, La Nuova Italia, 1975. 
ed è in qualche modo negativa, ha i tratti del peccato, perché comporta la corruzione della propria purezza. ${ }^{2}$ Sono aspetti sui quali il narratore ritorna a più riprese, in particolare nel passaggio che segue:

- Tu devi esser vigile affinché ciò ch'è già stato non muti. Ma ecco che se tu operi muti; tu non ti muovi senza che qualcosa in te, intorno a te non si muti; tu vivi, ed accresci e rimuti, accrescendo e mutando. Vedi dunque come per i delicati ed i timidi sia dolorosa l'azione. Perché vi sentono accanto il peccato; perché se operi, strappi, perché se operi rompi: opera e tu rompi la legge e il passato che ha deciso, che decide sempre di sé d'esser la legge. (E questo è lo sforzo, qui la lacerazione e lo sforzo e il dolore: a scindere dal Passato la Legge, a fissar la Legge qual sia nel molto passato). Or ecco che se tu vivi accresci e rimuti, tu operi e quando tu hai operato ecco che l'opera tua non è secondo il passato o secondo la legge: è peccato. Credi tu di poter vivere senza peccato?3

Pur manifestando una componente autobiografica (certo romanzata rispetto alle reali vicende dell'autore), l'opera «non si risolve in una autobiografia, ma in una biografia di idee, di emozioni oggettivate attraverso un processo di astrazione del confuso magma esistenziale-interiore». ${ }^{4} \mathrm{E}$ se presenta alcuni caratteri del romanzo di formazione - sentimentale e intellettuale - li risolve tuttavia in maniera antitradizionale: «l'inquietudine, il disordine, la contraddizione, sono il risultato dell'esperienza compiuta». ${ }^{5} \mathrm{Si}$ tratta nell'insieme di un'opera refrattaria alle catalogazioni, certamente «al di sopra di ogni sospetto ottocentesco», ${ }^{6}$ ma che "contiene, a blocchi, le molte maniere di raccontare che fra Otto e Novecento in Italia si sono affrontate e scontrate».?

La combinazione di narrazione e riflessione, con evidente predominanza della seconda ${ }^{8}$ si associa allo sdoppiamento del protagonista, che a di-

2 Sulla «contraddizione fra "ordine" e "peccato"» che articola l'opera nel suo complesso, sul peccato come «trasgressione rispetto alla norma, alla legge, alla tradizione», si veda in particolare Giorgio Bàrberi Squarotti, Forma e vita nel "Peccato" di Boine, in Franco Contorbia (a c. di), Giovanni Boine. Atti del convegno nazionale di studi. Imperia, 25-27 novembre 1977, Genova, Il melangolo, 198I, pp. 34I-55 (pp. 34I e 352 per i passaggi citati).

3 Boine, Il peccato, a c. di Davide Puccini, cit., p. 58. Per una prima ricognizione sulla lingua dell'autore si veda Gianfranco Contini, Alcuni fatti della lingua di Giovanni Boine, in Id., Varianti e altra linguistica, Torino, Einaudi, 1970, pp. 247-58.

4 Giulio Ungarelli, Nota introduttiva, in Boine, Il peccato, a c. di Giulio Ungarelli, cit., p. XI. Si veda anche Pier Giorgio Conti, Il "balbo narrare". Strutture e forme della confessione: una modalità del genere "romanzo biografico", «SIT!», 5, novembre 1996, pp. 159-67.

5 Bàrberi Squarotti, Forma e vita nel "Peccato" di Boine, cit., p. 345.

6 Ungarelli, Nota introduttiva, cit., p. VII.

7 Marziano Guglielminetti, "Il peccato" tra romanzo e autobiografia, in Contorbia (a c. di), Giovanni Boine. Atti del convegno nazionale di studi, cit., p. 363.

8 Si tratta di «una vicenda dove le idee, i pensieri, i commenti hanno la meglio sui fatti assun- 
stanza di qualche anno si fa narratore e commentatore della propria storia, con conseguente ambigua oscillazione fra riflessioni contemporanee ai fatti e riflessioni a posteriori. ${ }^{9} \mathrm{E}$ del resto «il vero punto centrale del discorso boiniano è rappresentato dalla scoperta della contemporaneità delle due posizioni nel protagonista del romanzo, ovvero dello sdoppiamento del protagonista stesso fra il custode della tradizione e il trasgressore». ${ }^{10}$

Alle riflessioni del protagonista si associano inoltre i commenti - solitamente molto brevi - di altri personaggi (un insieme generico di concittadini, più o meno esteso a seconda dell'oggetto del commento: il protagonista stesso, i giovani in generale, l'umanità).

Sul piano espressivo, questa combinazione di pensieri e punti di vista si manifesta in particolare attraverso l'impiego massiccio di forme incidentali, spesso segnalate da lineette e parentesi (soprattutto tonde, ma in due casi quadre); altre riflessioni e commenti sono invece segnalati da virgole, connettivi polifunzionali, espressioni di vario genere, e possono anche presentarsi come proposizioni indipendenti. Un censimento completo delle occorrenze di lineette e parentesi, incrociato ad uno spoglio dei casi di commento, potrebbe consentire l'individuazione di alcune linee di tendenza. La varietà delle potenzialità espressive di questo innesto testuale dispiegata nel Peccato, fortemente dipendente dall'interpretazione, suggerisce tuttavia di circoscrivere almeno per il momento l'analisi a una selezione di estratti che presentano una tipologia diversificata di innesti metatestuali, individuando alcune peculiarità.

La proposta si inserisce nel quadro di una ricerca di linguistica testuale volta a esaminare il funzionamento delle porzioni testuali che si riferiscono ad altro testo (con osservazioni che concernono la forma o il contenuto, oppure entrambi), e in particolare a individuare e classificare le forme della nota in rapporto alle modalità di autocommento, attraverso una catalogazione basata sull'aspetto più immediatamente percepibile, la collocazione spaziale. A un primo livello si ha il commento integrato alla narrazione, che può avere la forma di una proposizione indipendente (o di un capoverso),

ti esclusivamente in funzione di pretesto-occasione del discorso narrativo» (Ungarelli, Nota introduttiva, cit., p. XI).

9 La vicenda è narrata in terza persona, con passaggi alla seconda persona in genere connessi ad una dimensione più spiccatamente riflessiva («Egli e tu si rivelano un’immagine riflessa dell'io, anche se tale immagine pare avere più consistenza della figura da cui trae origine», Davide Puccini, L'opera, in Boine, Il peccato, a c. di Davide Puccini, cit., p. XXXIV).

IO Bàrberi Squarotti, Forma e vita nel "Peccato" di Boine, cit., p. 353. Per un'approfondita analisi dei rapporti fra narratore e personaggio si veda Walter Breitenmoser e Clà Riatsch, "Questo aggroviglio immane": distanza e vicinanza tra narratore e personaggio nel "Peccato" di Boine, «SIT!», 5, novembre 1996, pp. I69-82. Va osservato che il gioco di specchi fra protagonista e narratore si trasmette anche all'autore: anonimo, il narratore viene chiamato ad un certo punto «signor B.» da suor Maria (Boine, Il peccato, a c. di Davide Puccini, cit., p. 32), ed è definito «giovanotto di ventisei anni» (ibidem, p. 42), età dell'autore al momento della stesura del testo. 
oppure presentarsi come inciso. Ai livelli successivi si collocano le note a piè di pagina, le note finali con rinvio puntuale, le note introduttive o di chiusura, e infine le note indipendenti collocate su supporti diversi da quello del testo principale. ${ }^{I I}$

Il caso del Peccato si situa felicemente in questo panorama, ma richiede due fondamentali precisazioni. In primo luogo, non si tratta di commento metalinguistico: non ci situiamo dunque nel contesto delle glosse - esplicative o eventualmente digressive - che fanno riferimento alla forma di quanto precede, alle parole impiegate, al linguaggio scelto (strategia tipica della prosa di Gadda o di Meneghello, in particolare). Con Boine abbiamo un tipo di commento che si rivolge essenzialmente ai contenuti, rielaborandoli, completandoli e valutandoli. ${ }^{12}$

Secondariamente, la metatestualità del Peccato si concentra interamente nel primo livello indicato, quello del commento integrato alla narrazione: non vi sono infatti note distinte spazialmente dal testo principale. L'interesse si situa dunque a livello dell'analisi delle diverse modalità di integrazione del metatesto, in parte segnalate da parentesi o lineette, in parte da altri segnali interpuntivi o particolari configurazioni linguistiche.

Proposte parallele da parte di un diverso io, ${ }^{13}$ pause nel discorso narrativo, ${ }^{14}$ le parentetiche nel Peccato presentano funzioni diversificate e non sempre univoche, che possono anche essere intese globalmente come risvolto

II Una prima proposta in questo senso è esposta nella mia tesi di dottorato, discussa il 3I gennaio 2015 e pubblicata l'anno successivo (Sveva Frigerio, Linguistica della nota. Strategie metatestuali autoriali, Ginevra, Slatkine, 20I6). Per una sintesi degli aspetti chiave della classificazione, corredata di esempi letterari, si veda Strategie metatestuali autoriali, in Lettura e edizione di testi italiani (secc. XIII-XX). Dieci progetti di dottorato di ricerca all'Università di Ginevra, a c. di Massimo Danzi, "Quaderni ginevrini di italianistica", n. 2 (20I4), pp. 35I-4I5.

I2 I commenti nel Peccato tendono anzi a oscillare fra dimensione intratestuale ed extratestuale. Si sono naturalmente privilegiati in questa sede i casi appartenenti alla prima, i soli effettivamente riconducibili alla categoria della metatestualità delineata nei lavori citati (si veda la nota precedente).

I3 «Il protagonista, a mano a mano che il romanzo procede, vede sempre più vivamente e intensamente presente il suo doppio, che le parentetiche sottolineano nella funzione di proposta non alternativa, ma contemporanea di procedimenti, idee, comportamenti, pensieri, considerazioni opposte rispetto a quelle che il protagonista presenta e propone» (Bàrberi Squarotti, Forma e vita nel "Peccato" di Boine, cit., p. 346). Sull'effetto di eterogeneità enunciativa introdotto dalle parentetiche si veda in particolare Luca Cignetti, Le parentesi tonde: un segno pragmatico di eterogeneità enunciativa, in Angela Ferrari (con Luca Cignetti, Anna-Maria De Cesare, Letizia Lala, Magda Mandelli, Luciano Zampese), La lingua nel testo, il testo nella lingua, Torino, Istituto dell'Atlante Linguistico Italiano, 2004, pp. I65-89, e il successivo volume di Luca Cignetti, Linciso. Natura linguistica e funzioni testuali, Alessandria, Edizioni dell'Orso, 201 .

I4 La scrittura di Boine, «essenzialmente parentetica, tende a dare l'equivalente di un parlato, sia interiore che esterno, anche mediante la ripetizione senza soluzione di continuità di diversi segni d'interpunzione e con l'uso frequentissimo delle lineette, non per isolare un inciso, ma per segnare una pausa del discorso narrativo» (Ungarelli, Nota al testo, cit., p.XXV). 
formale del flusso di coscienza, in una sua precoce rappresentazione. $\mathrm{E}$ in tale direzione indirizza forse lo stesso Boine, che recensendo Faville di Mario Puccini scrive: «vedo che anche lui usa le parentesi-sottovoci, a introdurre i ragionamenti in margine, i soliloqui-cuneo come qualche altro giovane scrittore di mia conoscenza».. ${ }^{15}$

Si veda innanzitutto il seguente passo, tratto dai paragrafi iniziali, dove le parentesi accolgono pensieri e commenti altrui, volti essenzialmente a evocare la condizione di isolamento dell'intellettuale, incompreso e giudicato dalla società:

S'eran sentite certe voci su lui di quand'era agli studi... E par che avesse detto ch'egli al catechismo nelle scuole non ci teneva gran che. - Pei politicanti del Consiglio comunale egli era un «originale». Non si capiva cos'era. Aveva scritto sul giornale del sito in pro, che so io, della «scuola serale» (dunque è con noi socialisti) e poi detto male del discorso del tale e del talaltro al comizio del primo maggio passato (dunque non è socialista). Si mescolava del resto di rado nelle conversazioni a caffè; non giocava; che avesse donne nessuno per allora sapeva; le compagnie allegre, quelle che restan di notte fino alle due in schiamazzi a far la serenata alla bella, o si spandon fra le quinte in teatro l'inverno a pizzicar le coriste, i giovanotti che capiscon la vita e come si deve («son nell'età!») se la godono, quelli lo avevano un poco in concetto tra di «prete» e babbeo. ${ }^{16}$

Le parentesi accolgono nei primi due casi le conclusioni dei politicanti circa l'essere o meno socialista del protagonista, che confrontate al commento posto fra virgolette («originale») fanno supporre che si tratti di sintesi, di generalizzazioni rispetto a discorsi più ampi o ripetuti in più occasioni. La terza parentesi accoglie un commento racchiuso tra virgolette e tra parentesi, attribuibile ad una cerchia più larga di cittadini, e non solo ai politicanti; i segni tipografici danno anzi l'impressione che l'autore voglia isolare più nettamente quella che appare come una sentenza di carattere generale.

Poco più avanti, descritti alcuni amici, il narratore scrive:

S’era dunque fatti amici di gente siffatta, e faceva con essi vita in disparte,

I5 Boine, Plausi e botte, in Id., Il peccato, a c. di Davide Puccini, cit., p. 158. Fra i modelli di Boine, oltre alle Confessioni di Sant'Agostino, vi è il Libro de la vida di Teresa de Avila, «dove la forma diretta e quella indiretta si scambiano continuamente di posto, dove un particolare, un ricordo, richiamati da una riflessione interrompono il filo del pensiero, ma per arricchirlo di una sostanza corporale oppure, nel caso inverso, dove il racconto vero e proprio è interrotto da ampi commenti-digressioni che ne legittimano la specifica qualità esemplare» (Ungarelli, Nota introduttiva, cit., p. XIII).

I6 Boine, Il peccato, a c. di Davide Puccini, cit., pp.3-4. Un'analisi più ampia di questo estratto e dei tre successivi è proposta anche in Frigerio, Linguistica della nota, cit., pp. 295-300, tuttavia con significative differenze determinate dalle esigenze dell'insieme. 
specie di chiusa, malnota fratrìa nella affacendata materiale quietudine [...] della vecchia sbadigliante città. (Oh! notturne escursioni in disputante frotta su per i vicoli zitti del dormiente paese! Oh! amicali libazioni di bianco vino sulla terrazza nota, come un alto sperone sul mare e discussioni focose! Oh! esoterici conciliaboli e sguardi diffidenti della gente d'intorno). Stava in disparte con essi $\left[. . .{ }^{17}\right.$

In questo caso una lunga parentetica composta da una successione di esclamazioni viene posta fra due frasi compiute (segue cioè una frase chiusa da un punto fermo e precede una maiuscola). Le occupazioni della malnota fratria sono messe in risalto dalla parziale corrispondenza sintattica delle tre proposizioni nominali, che in apertura, subito dopo l'esclamazione, collocano il sintagma nominale, sempre con l'aggettivo a precedere il sostantivo (notturne escursioni, amicali libazioni, esoterici conciliaboli), seguito poi dalle diverse specificazioni o aggiunte. La celebrazione dei piaceri sarà un commento del narratore (che ricorda il passato) o eventualmente del protagonista (che celebra il presente), con il compiacimento dato dal sentimento di superiorità rispetto al dormiente paese e agli sguardi diffidenti della gente d'intorno. ${ }^{18}$

Fra i commenti del narratore che si pongono al di sopra di quelli della gente, e che sono a seconda dei casi più o meno distinguibili da quelli del protagonista, oltre al precedente (e a manifestazioni del tutto implicite, come forse il politicanti del primo estratto), vi sono casi ben più chiari, come quello della parentetica seguente, che si trova poche righe dopo l'estratto appena citato:

Stava in disparte (grettezza maligna di una città di provincia e contrasti grotteschi; piccineria con mille occhi stupidamente curiosi su te, se ci capiti in mezzo!) come non degnandosi, dicevano; e dentro si capisce la gente se ne sentiva offesa un poco. ${ }^{19}$

Il passaggio consiste in un giudizio della gente (Stava in disparte [...] come non degnandosi), e forse, più precisamente, ancora dei politicanti citati nel primo estratto; il commento è qualificabile come tale solo in un secondo tempo (grazie a dicevano), e il termine in corsivo (degnandosi) evidenzia la forma

I7 Boine, Il peccato, a c. di Davide Puccini, cit., p. 5.

I8 I meccanismi di ripresa dopo una pausa di commento (o, come in questo caso, un'espansione descrittiva) rappresentano uno degli aspetti più complessi dell'introduzione di un secondo livello discorsivo; al rischio di perdere il filo del discorso, l'autore spesso supplisce con una ripresa identica o una riformulazione. In questo caso, la ripresa avviene per inversione dei complementi e sostituzione del verbo fare con stare (faceva con essi vita in disparte > stava in disparte con essi).

I9 Ibidem, p. 6 (corsivo dell'autore). 
diretta del discorso riportato. Il commento include un ulteriore commento, questa volta del narratore, isolato all'interno di un inserto parentetico e di carica fortemente soggettiva (grettezza maligna, contrasti grotteschi, piccineria, stupidamente), in cui ci si riferisce ad un $t u$ indefinito (identificabile nuovamente con il narratore, analogamente al primo estratto riportato). Il narratore passa poi ad una sorta di onniscienza (il testo, fortemente incentrato sull'io, manifesta raramente tale slittamento), per commentare il sentimento della gente, con una forma verbale (si capisce) che funge da rafforzativo del commento e al contempo lo motiva.

Un caso in cui il passaggio dal commento del protagonista al commento del narratore appare più netto è il seguente:

E se una questione seria niente niente, era agitata in città, non in farmacia dove si fan chiacchiere e non servono, ma sul giornale le sue opinioni nette (credeva, e c'era del buffo in questo suo ingenuo illudersi. Quanta illusione, amici! E come tuttociò che è netto per noi tutti questi altri lo piglian per nebbia!) da offendere sino, non si faceva pregare a metterle fuori. ${ }^{20}$

In questo caso nell'intervento narratoriale tra parentesi ci sono al tempo stesso indici di distanza del narratore dal personaggio (credeva, suo ingenuo illudersi) e di vicinanza (noi). ${ }^{21}$ L'utilizzo della prima persona plurale tende inoltre a includere il lettore, per empatia, nella condizione dell'intellettuale delineata dall'autore attraverso la rappresentazione di se stesso.

Proseguendo nella lettura, risulta più evidente la presenza di enunciati parentetici che diversamente da quelli visti finora non commentano in senso stretto il testo, ma completano determinati aspetti concreti della vicenda (ad esempio precisano le descrizioni), ricorrendo ai ricordi, rielaborati a posteriori, ed eventualmente anche a ipotesi. È quanto accade in questo passaggio (una descrizione notturna che prelude a un incontro clandestino fra gli innamorati):

Salire su ai lecci era facile (si rizzò agile e rapido. E come, scavalcato il muro sentì sotto i piedi, qua, là il tondeggiar delle ghiande si chinò come da bimbo pronto ridente, tastando, a raccoglierne) ma sportosi all'altra cinta di fra i cocci di vetro a fatica, si vedevan giù bassi come in un pozzo i solchi soffici uguali ed i cespi d'ortaglia. Era diffusa per l'aria una freschezza chiara (forse

20 Ibidem, p. 6.

2I È stato osservato perciò che il testo propone "un personaggio "ingenuo" al momento della storia che si illude che gli altri lo comprendano, e un personaggio "saggio" al momento della rimemorazione in cui il suo punto di vista coincide con quello del narratore. L'interiezione: "Quanta illusione amici!" che connota l'aspetto doloroso della perdita delle illusioni può valere anche come indizio del sostrato omodiegetico del racconto» (Breitenmoser e Riatsch, Questo aggroviglio immane, cit., p. I76). 
si levava all'orizzonte la luna) e veniva dal mare, il rumore dell'onda un po' mossa. I lecci stavano immobili, scuri di sopra il suo capo (senza stormire); un odore di terra umidiccia e di muschio si levava d'intorno; una finestra di là dal convento alle case del ponte, rossa in una gran chiazza scura, luceva come a fissarlo; a sinistra più in alto, indecisa la massa enorme, contro il cielo stellato, del già dormente paese colle rade accese collane, (due, tre, parallele) dei fanali giallicci. Il convento era zitto e tutto chiuso: di là dei suoi tetti sghembi e del campaniletto a punta forato, sentivi l'ondoso allargarsi del mare. Attese. ${ }^{22}$

Vi sono quattro parentetiche in poche righe, la prima all'interno di un passaggio che oscilla fra narrazione e descrizione, le successive inserite nella descrizione vera e propria. La parentesi più ampia e complessa è la prima, spezzata al suo interno da un punto fermo, che divide due segmenti sostanzialmente indipendenti l'uno dall'altro (il protagonista sale agile $e$ rapido, poi scavalca il muro e si china come da bimbo a tastare e raccogliere le ghiande). A sua volta il testo principale è indipendente dalla parentesi, e il connettivo oppositivo $m a$ che segue l'innesto, pur implicando una lettura interpretativa, non sembra coinvolgerlo (la salita è facile, ma conduce molto in alto, tanto da vedere giù bassi come in un pozzo i solchi soffici uguali ed $i$ cespi d'ortaglia). La seconda parentesi introduce un'ipotesi formulata a posteriori (il ricordo è carente: forse c'era la luna), che vorrebbe completare la descrizione, motivare il fatto che la freschezza sia chiara. ${ }^{23}$ La terza rafforza a distanza l'aggettivo immobili, senza sembrare ulteriormente fondata (forse vuole sottolineare che i lecci non stormiscono, nonostante sul mare l'onda sia un po' mossa). La quarta indica numero e disposizione delle accese collane dei fanali giallicci (con approssimazione, a indicare un progressivo precisarsi della percezione nel passato, o di nuovo un ricordo carente nel presente).

È particolare anche la modalità descrittiva, anticipata dal più canonico dei verba videndi (si vedevan), ma aperta da altre percezioni sensoriali, tattili (la freschezza dell'aria) e uditive (il rumore dell'onda un po' mossa), mentre quelle visive sono date dal volgersi in direzione opposta a quella precedentemente indicata in relazione al verbo (i solchi e $i$ cespi si vedono giù, mente $i$ lecci sono sopra il suo capo).

Meccanismi ugualmente diversificati si riscontrano più avanti (il salto nel buio per raggiungere la suora ha comportato una ferita al braccio, che la giovane si presta prontamente a curare):

Lo fece sedere su di un tronco abbattuto; sedette accanto chinata (c'eran

22 Boine, Il peccato, a c. di Davide Puccini, cit., pp. 38-39.

$23 \mathrm{Ma}$ è anche eco pascoliana, quasi il narratore rispondesse - con cautela, senza certezze alla domanda che apre L'assiuolo (Myricae): «Dov'era la luna?» (v. I). E la freschezza chiara della notte rinvia all'«alba di perla» (v. 2) e alla «nebbia di latte» (v. Io) del testo pascoliano. 
dietro loro a spalliera dei gran cespi di vaniglia odorosa) e fasciava attenta e stringeva. Zitta... Era una donna, una donna... Sentivi sotto l'abito lieve l'ansimare del petto; le ciocche abbondanti ai lati del viso in due bande giù; la radice del collo bianca (nuda) fin giù, lineata, allo scuro dell'abito; e (gli pareva), s'ella alzava lo sguardo un rilucere vivo negli occhi... era una donna, una donna... Ma com'ebbe finito, si scosse, sembrò smarrita, volle muoversi, alzarsi: la tenne. «E come risale ora?» chiese spaurita. «Risalirò». I muri tutt'intorno (umidore nero e cespugli), cingevano altissimi; da un lato, pesanti, i cipressi ed i lecci (enormi); dall'altro a mare ombrosi il campanile ed indeciso il convento (accosciato, come una gran bestia torpidamente dormiente); su, brillanti pungenti d'argento, nel cavo le stelle. ${ }^{24}$

A una prima parentesi descrittiva (improntata alla dimensione olfattiva molto più che a quella visiva, che del resto è fortemente limitata dall'oscurità) ne segue una seconda, dove la descrizione è in realtà un'esplicitazione che accentua la sensualità del dettaglio (la radice del collo, bianca, è anche nuda). La terza segnala la soggettività della percezione (gli pareva, che vi fosse un rilucere vivo negli occhi della suora). Le successive arricchiscono le percezioni: umidore nero e cespugli caratterizzano $i$ muri tutt'intorno, che sono detti successivamente altissimi nel testo principale, e la grandezza degli elementi del paesaggio è ulteriormente accentuata nelle due ultime parentesi (cipressi e lecci sono enormi, e il convento è associato per similitudine a una gran bestia).

Altri casi ancora presentano tra parentesi una vera e propria alternativa, senza esplicitazione del legame con il testo principale. In una frase come "Disse calma (fredda) "Tuo zio Battista è morto"», ${ }^{25}$ può trattarsi di un'interpretazione del narratore, determinata dalle circostanze (lo scandalo ha guastato i rapporti del narratore con la madre, che gli si rivolge con freddezza).

Le quasi duecentocinquanta parentesi presenti nel testo manifestano insomma - lo si è visto già in questi pochi casi - una grande varietà di funzioni, rispetto alle quali l'interpretazione riveste un ruolo importante. A complicare il quadro concorrono casi in cui, magari a brevissima distanza, vi sono formule che pur presentando potenzialmente identica funzione in un caso si inseriscono fra parentesi, mentre in un altro sono poste fra virgole correlative: sarà quindi il segno stesso a diversificare il rilievo.

Per quanto riguarda gli impieghi delle lineette (ve ne sono più di un centinaio nel testo), possono essere analoghi a quelli delle parentesi: si avrà in questo caso una coppia di lineette, dove la seconda, diversamente dall'indispensabile parentesi che chiude, può essere assorbita da un segno di interpunzione più forte, come il punto o il punto e virgola. 
L'inserimento di segmenti di discorso diretto privi di introduttori sintattici, come nel caso seguente, è una delle funzioni che accomuna lineette e parentesi (nel Peccato il discorso diretto è marcato anche da virgolette basse, con conseguente possibile moltiplicazione dei segni tipografici):

Ma la conclusione di chi leggeva essendo poi sempre la stessa - «ma con chi è? ma cos'è dunque lui per suo conto?» - aveva concluso ch'era meglio star zitti in disparte. ${ }^{26}$

Vi sono anche casi in cui le lineette accolgono integrazioni chiarificatrici, come nel passaggio seguente, riferito ai giovani:

Son fuori del mondo, l'han tutto innanzi e di fuori, - non l'hanno ancora creato - e perciò come falsi iddii, manovrando con miti illusori di male e di bene, sono a vuoto severi e lo giudicano. ${ }^{27}$

In alcuni casi tuttavia l'impiego della lineetta manifesta particolare originalità, abbandonando lo schema incidentale:

Passione, passione, passione: l'ira e l'amore, la tortura e l'amore, la gelosia e l'amore, lo scherzare lieve e l'amore, l'amore e il dolore, la melanconia dolce e la gioia chiassosa, la leggerezza e la burla, la vendetta e l'inganno, e l'amore e l'amore e poi sempre l'amore. (E senti dunque come in questa voce vive la carne, come dice, come dice, senti come dice e commuove, - lascia il tuo cuore tremare, - lasciati via d'un tratto portare e commuovere - senti che forza reale di vita e che sentimentale umana passione!) Ascoltava. ${ }^{28}$

Non si tratta chiaramente di un impiego canonico della lineetta, quanto dell'evidenziazione atipica degli ultimi elementi di una climax racchiusa fra parentesi e articolata lungo una sequenza di imperativi (senti, senti, lascia, lasciati, senti), gli ultimi dei quali seguono immediatamente le tre lineette, invitando al trasporto emotivo; sembrerebbero quasi battute successive di una stessa voce.

I casi più frequenti sono tuttavia quelli in cui la lineetta si presenta con una singola occorrenza senza che sia plausibile un assorbimento del suo corrispettivo da parte di un segno più forte in chiusura: si tratta di casi in cui la lineetta si colloca a inizio frase, spesso segnalando, sembrerebbe, il passaggio al pensiero del narratore:

26 Ibidem, p. 6. Un caso analogo è «son nell'età!», posto fra parentesi (secondo estratto citato in questo contributo).

27 Ibidem, p. 8.

28 Ibidem, p. 67. 
Ed il nonno certo, arguto vecchietto tutto legge e latino, non s'era impacciato mai né di sfumature soggettivistiche novalisiane né di Sigismondi spagnuoli. Diceva ciò senza troppa tragedia. - Nemmeno lui per altro amava granché la tragedia. E non approvava. Bisognava pure accettare la vita. Ed il sogno era il sogno. ${ }^{29}$

Il meccanismo potrebbe essere ricondotto alla distinzione, propria di molta narrativa, fra discorso dialogato (posto fra virgolette basse) e discorso pensato (posto fra lineette), nelle varie forme che quest'ultimo può assumere, fra monologo interiore e flusso di coscienza. ${ }^{30}$

Nel complesso, per quanto riguarda l'introduzione di commenti, riflessioni, precisazioni, Boine si avvale spesso di procedimenti tipografici molto espliciti (virgolette, lineette, parentesi), ma può anche distinguere gli innesti dando loro forma di frase indipendente, o viceversa integrarli quasi completamente al testo, così da creare, nei commenti che più gli stanno a cuore, una maggiore vicinanza fra narrazione e intervento metatestuale, accordando così al proprio punto di vista una via d'accesso privilegiata al lettore, e fornendo una prova precoce e originale dell'impiego del flusso di coscienza.

Sveva.Frigerio@unige.ch

29 Ibidem, p. II. È stato osservato che in esso la sentenza e la tautologia «hanno un evidente valore gnomico in cui l'imperfetto, nel caso della tautologia crudamente ridondante, non ha altra funzione che segnalare il DIL» (Breitenmoser e Riatsch, Questo aggroviglio immane, cit., p. 172). Mi sembra tuttavia che si tratti piuttosto di una messa in discussione, tramite scomposizione e ricomposizione, del titolo del dramma di cui il Sigismondo appena citato è protagonista: La vita è sogno (163I-35) di Pedro Calderón de la Barca.

30 Particolarmente suggestive le considerazioni di Adorno a proposito della lineetta, con la quale «il pensiero si rende conto del suo carattere frammentario» (Theodor Adorno, Interpunzione, in Note per la letteratura. 1943-196I, trad. it. di Enrico De Angelis, Torino, Einaudi, 1979, p. I03; per il testo originale si veda Satzzeichen (1956), in Id., Noten zur Literatur. Gesammelte Schriften, vol. II, a c. di Rolf Tiedelmann, Frankfurt am Main, Suhrkamp, 1997, pp. I06-II3). Aggiunge Adorno che «la sensibilità dello scrittore nell'interpunzione si conferma nel modo di trattare gli incisi. Chi procede con prudenza inclinerà a porli fra due trattini e non in parentesi tonde poiché queste estrapolano completamente l'inciso dalla frase, creano per così dire delle enclaves mentre invece niente di ciò che avviene in una buona prosa dovrebbe essere superfluo alla struttura complessiva; confessando tale eluttabilità, le parentesi tonde rinunciano tacitamente a pretendere l'integrità della configurazione linguistica e capitolano davanti alla rozzezza pedantesca. Invece i trattini, che accantonano le frasi parentetiche dal flusso senza tenerle in prigioni, mantengono in eguale misura rapporto e distanza. Ma come la cieca fiducia nella loro forza di fare ciò sarebbe illusione perché si aspetterebbe dal semplice mezzo quel che può essere dato unicamente dalla lingua e dalla cosa stessa, così nell'alternativa di trattini e parentesi si può vedere quanto fragili sono le norme astratte dell'interpunzione» (ibidem, p. Io6). Il termine originale è lo splendido «Gedankenstrich», quasi "linea del pensiero", da rendere preferibilmente con "lineetta" (il termine "trattino" è una scelta del traduttore). 